\title{
Molecular-oxygen-promoted Cu-catalyzed oxidative direct amidation of nonactivated carboxylic acids with azoles
}

\author{
Wen Ding ${ }^{1}$, Shaoyu $\mathrm{Mai}^{2}$ and Quuling Song ${ }^{* 1,3}$
}

\author{
Full Research Paper \\ Address: \\ ${ }^{1}$ Institute of Next Generation Matter Transformation, College of \\ Chemical Engineering at Huaqiao Univeristy, P. R. China, ${ }^{2}$ College of \\ Materials Science at Huaqiao University, 668 Jimei Blvd, Xiamen, \\ Fujian, 361021, P. R. China and ${ }^{3}$ Beijing National Laboratory for \\ Molecular Sciences, Beijing, 100190, P. R. China \\ Email: \\ Qiuling Song* - qsong@hqu.edu.cn \\ ${ }^{*}$ Corresponding author \\ Keywords: \\ amidation; azoles; Cu-catalyzed; molecular oxygen; transamidation
}

Open Access
doi:10.3762/bjoc. 11.233
Received: 25 August 2015
Accepted: 26 October 2015
Published: 11 November 2015
This article is part of the Thematic Series "Copper catalysis in organic
synthesis".
Guest Editor: S. R. Chemler
C 2015 Ding et al; licensee Beilstein-Institut.
License and terms: see end of document.

\begin{abstract}
\section{Introduction}

Amides are prevalent scaffolds in numerous compounds ranging from biologically active natural products to pharmaceuticals [1]. As a result, numerous methods have been developed for the formation of amides [2-7]. Despite such advances [8-21], the acylation of amines with free carboxylic acids is still the most common method employed due to the availability of the starting materials and its relatively clean process feature, whereby water is the only byproduct in the transformation [22]. However, preactivation of the free carboxylic acids is always required, and stoichiometric activating reagents or coupling reagents are mandatory in the classic methods of amide formation (Scheme 1a) [23].
\end{abstract}

A copper-catalyzed oxidative direct formation of amides from nonactivated carboxylic acids and azoles with dioxygen as an activating reagent is reported. The azole amides were produced in good to excellent yields with a broad substrate scope. The mechanistic studies reveal that oxygen plays an essential role in the success of the amidation reactions with copper peroxycarboxylate as the key intermediate. Transamidation occurs smoothly between azole amide and a variety of amines.

In some cases, corrosive byproducts $(\mathrm{HCl})$ are unavoidable and the activating reagent is difficult to remove from the system.
Given the growing demand for environmentally benign synthesis [24], it is highly desirable to bring forth a green, sustainable and simple new protocol for the activation of carboxylic acids. Molecular oxygen is an ideal oxidant owing to its negligible cost, availability and sustainability $[25,26]$. Currently, copper-catalyzed, aerobic, oxidative transformations with dioxygen as the sole oxidant have emerged as a useful and powerful strategy to construct carbon-carbon and carbon-heteroatom bonds [27-29].

It is known that $\mathrm{Cu}$ (II) superoxide species are usually reported to be formed in the system, and very recently McDonald and coworkers reported an unusual nucleophilic reactivity of a special $\mathrm{Cu}$ (II) superoxide complex, acting as a deformylating reagent in spite of the prevailing electrophilic property common 


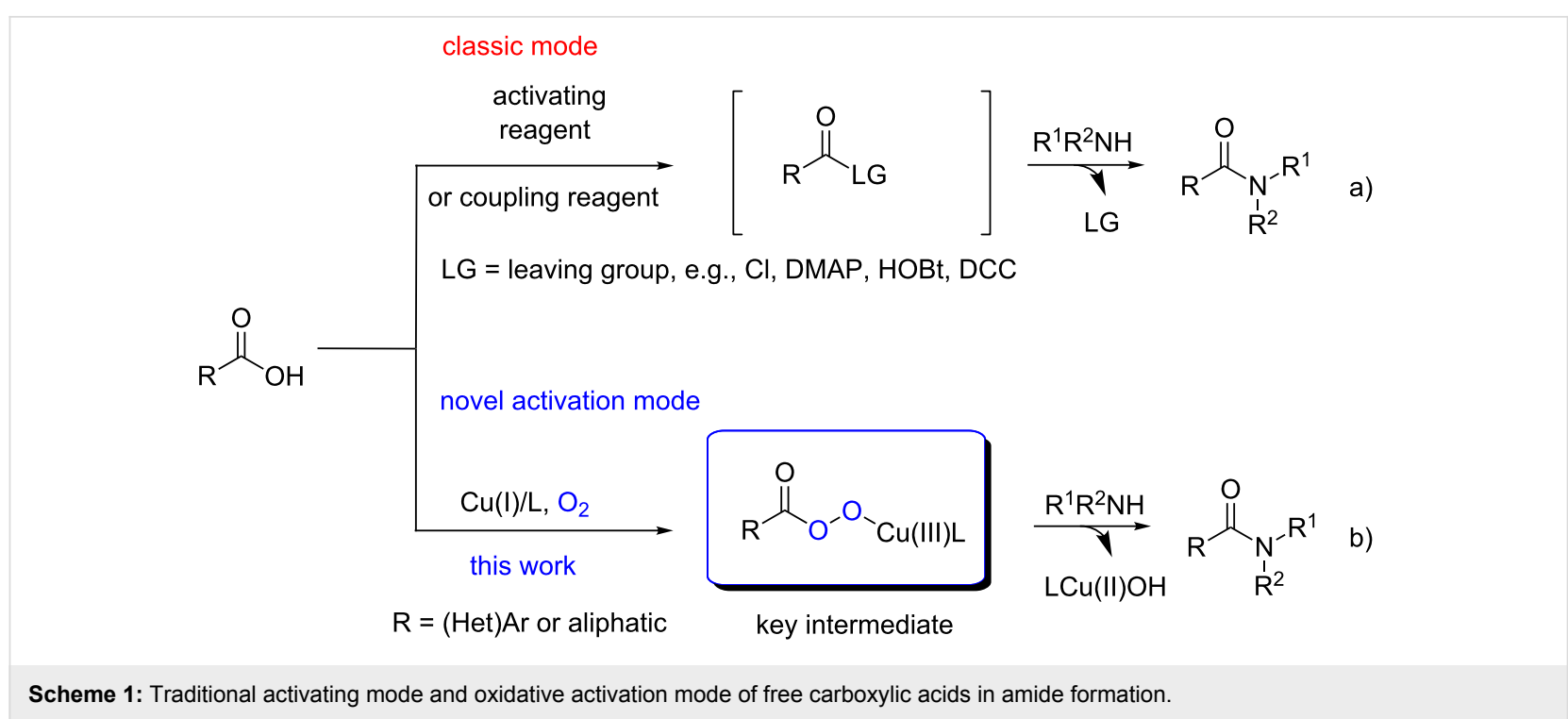

for such a metal-bound superoxide intermediate [30]. Therefore, we envision that the newly discovered nucleophilic reactivity of $\mathrm{Cu}$ (II) superoxide species might lead to a novel, free carboxylic acid activation mode for amide formation: a $\mathrm{Cu}(\mathrm{II})$ superoxide species could attack the carbonyl group in an acid to afford a copper peroxycarboxylate, which might then serve as the key intermediate to make the subsequent amide formation feasible (Scheme 1b). In our work, azoles have been chosen as the amines due to their special bioactivity [31]. To the best of our knowledge, $\mathrm{Cu}$ salt has not yet been used for catalyzed, oxidative direct amide formation. We report the first amidation reaction from carboxylic acids with peroxycarboxylate as the key intermediate, which represents a novel activation mode with molecular oxygen as the activating reagent. Most remarkably, in sharp contrast to previous reports (which used complex $\mathrm{N}$-containing ligands to form copper superoxide), inexpensive and readily available pyridine was employed as both the ligand and base in our case.

\section{Results and Discussion}

Our initial exploration commenced with benzoic acid (1) and benzimidazole (2) as the model substrates to investigate the copper-catalyzed oxidative direct amidation reaction (Table 1).

The copper salts demonstrated good activity for this novel transformation (Table 1, entries 1-5) and $85 \%$ of the desired product was obtained using $10 \mathrm{~mol} \%$ of $\mathrm{CuBr}$ with pyridine (3 equiv) at $130{ }^{\circ} \mathrm{C}$ in $p$-xylene $(1 \mathrm{~mL})$ under $\mathrm{O}_{2}$ in a sealed tube (Table 1 , entry 4 ). Notably, $\mathrm{CuBr}_{2}$ only gave $33 \%$ of the desired product under similar conditions (Table 1, entry 5), inferring that molecular oxygen was not just acting as an oxidant to convert $\mathrm{Cu}(\mathrm{I})$ into $\mathrm{Cu}(\mathrm{II})$, but that it might be involved in the reaction. Further screening of solvents and ligands revealed that $p$-xylene and pyridine are the optimal choices. The reaction cannot go to completion and part of the carboxylic acid remains when the amount of benzimidazole is not twice that of the carboxylic acid. The temperature affected this reaction dramatically and when the temperature was decreased to $120{ }^{\circ} \mathrm{C}$, the yield of the desired product 3 was reduced to $31 \%$ of the corresponding GC yield (Table 1, entry 14). Copper salt, pyridine and dioxygen were all found to be pivotal to this transformation (Table 1, entries 19-21): without $\mathrm{CuBr}$, no desired product was detected; in the absence of pyridine, the yield of the desired product was reduced to $46 \%$ with some unknown compounds generated; when the reactions were conducted under $\mathrm{N}_{2}$ atmosphere or air, the desired products were either not detected or reduced to $53 \%$.

With the optimized conditions in hand, we studied the scope and limitations of the $\mathrm{Cu}$-catalyzed oxidative direct amidation reaction. First, we surveyed different carboxylic acids. To our delight, both aromatic and aliphatic carboxylic acids were competent candidates in this transformation (Scheme 2).

For aromatic carboxylic acids, various substituents on the aromatic ring were tolerable under the standard conditions, which suggests that the reaction has a broad substrate scope. Electron-donating (4-9, 13, and 15-17) and electron-withdrawing groups $(\mathbf{1 0}-\mathbf{1 2}, \mathbf{1 4}, \mathbf{1 8}$, and 19) are also compatible in this reaction. Meanwhile, other aromatic acids such as 2-naphthylcarboxylic acid and pyrene-4-carboxylic acid also worked well under the reaction conditions, providing the desired products $\mathbf{2 0}$ and $\mathbf{2 1}$ in $59 \%$ and $88 \%$ yield, respectively. Additionally, heterocyclic acids, such as thiophene- and furan3-carboxylic acid were well tolerated, providing the amides 22 
Table 1: Optimization of the reaction conditions. ${ }^{\text {a }}$<smiles>O=C(O)c1ccccc1</smiles>

1<smiles>c1ccc2[nH]cnc2c1</smiles>

2

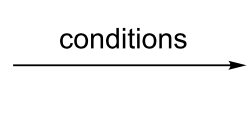

\begin{tabular}{|c|c|c|c|c|c|c|}
\hline entry & catalyst (mol \%) & atmosphere & ligand (equiv) & temperature & solvent & yield $(\%)^{b}$ \\
\hline 1 & CuCl (10) & $\mathrm{O}_{2}$ & pyridine (3) & $130^{\circ} \mathrm{C}$ & $p$-xylene & 33 \\
\hline 2 & Cul (10) & $\mathrm{O}_{2}$ & pyridine (3) & $130{ }^{\circ} \mathrm{C}$ & $p$-xylene & 79 \\
\hline 3 & $\mathrm{Cu}(\mathrm{OAc})_{2}(10)$ & $\mathrm{O}_{2}$ & pyridine (3) & $130{ }^{\circ} \mathrm{C}$ & $p$-xylene & 0 \\
\hline 4 & CuBr (10) & $\mathrm{O}_{2}$ & pyridine (3) & $130^{\circ} \mathrm{C}$ & $p$-xylene & $97(85)^{c}$ \\
\hline 5 & $\mathrm{CuBr}_{2}(10)$ & $\mathrm{O}_{2}$ & pyridine (3) & $130{ }^{\circ} \mathrm{C}$ & $p$-xylene & 33 \\
\hline 6 & CuBr (10) & $\mathrm{O}_{2}$ & pyridine (3) & $130{ }^{\circ} \mathrm{C}$ & o-xylene & 48 \\
\hline 7 & CuBr (10) & $\mathrm{O}_{2}$ & pyridine (3) & $130^{\circ} \mathrm{C}$ & DMSO & no product \\
\hline 8 & CuBr (10) & $\mathrm{O}_{2}$ & pyridine (3) & $130{ }^{\circ} \mathrm{C}$ & DMF & no product \\
\hline 9 & CuBr (10) & $\mathrm{O}_{2}$ & pyridine (3) & $130{ }^{\circ} \mathrm{C}$ & toluene & 4 \\
\hline 10 & CuBr (10) & $\mathrm{O}_{2}$ & 1, 10-Phen (3) & $130^{\circ} \mathrm{C}$ & p-xylene & no product \\
\hline 11 & CuBr (10) & $\mathrm{O}_{2}$ & 2, 2'-bipy (3) & $130^{\circ} \mathrm{C}$ & $p$-xylene & no product \\
\hline 12 & CuBr (10) & $\mathrm{O}_{2}$ & $\begin{array}{c}\text { 2-aminopyridine } \\
\text { (3) }\end{array}$ & $130{ }^{\circ} \mathrm{C}$ & $p$-xylene & no product \\
\hline 13 & CuBr (10) & $\mathrm{O}_{2}$ & $\mathrm{Et}_{3} \mathrm{~N}(3)$ & $130^{\circ} \mathrm{C}$ & $p$-xylene & no product \\
\hline 14 & CuBr (10) & $\mathrm{O}_{2}$ & pyridine (3) & $120^{\circ} \mathrm{C}$ & $p$-xylene & 31 \\
\hline 15 & $\mathrm{CuBr}(10)$ & $\mathrm{O}_{2}$ & pyridine (0.5) & $130^{\circ} \mathrm{C}$ & $p$-xylene & 39 \\
\hline 16 & $\mathrm{CuBr}(10)$ & $\mathrm{O}_{2}$ & pyridine (2) & $130^{\circ} \mathrm{C}$ & $p$-xylene & 77 \\
\hline $17^{\mathrm{d}}$ & CuBr (10) & $\mathrm{O}_{2}$ & pyridine (3) & $130^{\circ} \mathrm{C}$ & $p$-xylene & 7 \\
\hline 18 & CuBr (10) & $\mathrm{N}_{2}$ & pyridine (3) & $130{ }^{\circ} \mathrm{C}$ & $p$-xylene & no product \\
\hline 19 & CuBr (10) & air & pyridine (3) & $130^{\circ} \mathrm{C}$ & $p$-xylene & 53 \\
\hline 20 & - & $\mathrm{O}_{2}$ & pyridine (3) & $130^{\circ} \mathrm{C}$ & $p$-xylene & no product \\
\hline 21 & $\mathrm{CuBr}(10)$ & $\mathrm{O}_{2}$ & - & $130^{\circ} \mathrm{C}$ & p-xylene & 46 \\
\hline
\end{tabular}

aReaction conditions: all reactions were performed with a mixture of $1(0.25 \mathrm{mmol}), 2(0.5 \mathrm{mmol}), \mathrm{Cu}$ salt $(10 \mathrm{~mol} \%)$, ligand, solvent (1 mL), $16 \mathrm{~h}$, temp., corresponding atmosphere. ${ }^{b} \mathrm{GC}$ yield. ${ }^{\mathrm{c}}$ Isolated yield. ${ }^{\mathrm{d}} \mathbf{1}(0.25 \mathrm{mmol}), 2(0.30 \mathrm{mmol})$.

and 23 in acceptable yields. Gratifyingly, aliphatic carboxylic acids, such as cyclohexanecarboxylic acid, hexanoic acid and acetic acid were all proven to be good substrates in this transformation and the desired products 24, 25 and $\mathbf{2 6}$ were obtained in reasonable yields. Moreover, bis-substituted benzimidazole also worked very well under the standard conditions to give the desired product 27 in $89 \%$ yield.

The scope of amine was further investigated and it seems that only benzimidazole and its derivatives were good candidates in the above direct amide formation (see Scheme S1 in Supporting Information File 1 for details). However, remarkably, when 2-aminopyridine (28) and benzimidazole (2) were mixed with benzoic acid under the standard conditions, compound $\mathbf{2 9}$ was obtained in $45 \%$ yield instead of the predicted amide 3 (Scheme 3a). Compound 3 was not detected in the reaction. Similarly, when 2-naphthoic acid (30) was used instead of benzoic acid (1), compound 31 was afforded in 56\% yield without formation of compound $\mathbf{2 0}$ (Scheme 3b). However, when a control experiment was conducted without benzimidazole (2) (Scheme 3c), compound 29 was not detected and the reaction resulted in a complex mixture of undesired compounds.

In order to determine the possibility of transamidation between compound $\mathbf{3}$ and 2-aminopyridine (28), azole amide $\mathbf{3}$ was mixed with 2-aminopyridine (28) under the standard conditions. Surprisingly, the azole amide $\mathbf{3}$ was transformed into $\mathbf{2 9}$ in $92 \%$ yield, which clearly demonstrated that transamidation occurred in this process (Scheme 4).

Further exploration into a variety of amines suggested that transamidation could be efficiently accomplished in the absence of the copper catalyst, pyridine and the versatile amines, including aromatic (29, 31-34, 36 and 37) and aliphatic (35, 38-40), primary $(29,31,34,37$ and 40$)$ and secondary $(32,33$, 35, 36, 38 and 39). Even the amino group as a natural product (methyl L-leucinate hydrochloride, 40) was compatible under the conditions, affording corresponding amides in good to 


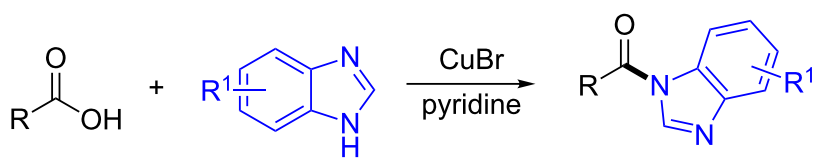<smiles>[R]c1ccc(C(=O)n2cnc3ccccc32)cc1</smiles>

3, $\mathrm{R}=\mathrm{H}, 85 \%$

$4, \mathrm{R}=\mathrm{Me}, 75 \%$

5, $\mathrm{R}=\mathrm{Et}, 92 \%$

6, $\mathrm{R}=\mathrm{iPr}, 71 \%$

7, $\mathrm{R}=n-\operatorname{Pr}, 64 \%$

8, $R=t-B u, 95 \%$

9, $\mathrm{R}=n-\mathrm{Bu}, 67 \%$<smiles>O=C(c1ccc(F)cc1)n1cnc2ccccc21</smiles>

$10,53 \%$<smiles>O=C(c1ccc(Cl)cc1)n1cnc2ccccc21</smiles>

$11,52 \%$<smiles>O=C(c1ccc(Br)cc1)n1cnc2ccccc21</smiles>

$12,40 \%$<smiles>COc1ccc(C(=O)n2cnc3ccccc32)cc1</smiles>

$13,77 \%$<smiles>Cc1ccccc1C(=O)n1cnc2ccccc21</smiles>

16, $76 \%$<smiles>O=C(c1cccc(Cl)c1)n1cnc2ccccc21</smiles>

$19,51 \%$<smiles>O=C(c1ccsc1)n1cnc2ccccc21</smiles>

22, $40 \%$<smiles>CC(=O)n1cnc2ccccc21</smiles>

26, $46 \%$<smiles>C=Cc1ccc(C(=O)n2cnc3ccccc32)cc1</smiles>

$14,89 \%$<smiles>O=C(c1ccc(C2CCCCC2)cc1)n1cnc2ccccc21</smiles>

$18,28 \%$<smiles>[R16]c1cc2c3ccc4cccc(c1)c4-c(=c-2C(=O)n1cnc2ccccc21)C=CC=3</smiles><smiles>O=C(C1CCCCC1)n1cnc2ccccc21</smiles>

$24,51 \%$<smiles>CCCCCC(=O)n1cnc2ccccc21</smiles>

$25,47 \%$<smiles>Cc1ccc(C(=O)n2cnc3ccccc32)cc1C</smiles>

$17,72 \%$<smiles>O=C(c1ccc2ccccc2c1)n1cnc2ccccc21</smiles>

$20,59 \%$

23, $61 \%$<smiles>Cc1cc2ncn(C(=O)c3ccccc3)c2cc1C</smiles>

$27,89 \%$

Scheme 2: Substrate scope for catalytic, direct amide formation from carboxylic acids and azoles. Reaction conditions: carboxylic acid ( $0.25 \mathrm{mmol})$, azole derivative $(0.5 \mathrm{mmol}), \mathrm{CuBr}(10 \mathrm{~mol} \%)$, pyridine $(0.75 \mathrm{mmol}), p$-xylene $(1 \mathrm{~mL}), 130{ }^{\circ} \mathrm{C}, 20 \mathrm{~h}$, under $\mathrm{O}_{2}$ atmosphere in a sealed tube. Isolated yield based on carboxylic acids.

excellent yield (Scheme 5), suggesting the potential utility in complex molecule manipulation. In addition, for the carboxylic acid part, both aromatic and aliphatic (37) compounds were competent as well.

The amidation reaction could be easily scaled up without significant decrease in the yield (Scheme 6).
Radical trapping experiments were performed in order to gain further insight into the mechanism of the catalytic direct amide formation from nonactivated carboxylic acids. The reactions were totally inhibited by radical scavengers 2,2,6,6-tetramethyl1-piperidinyloxy (TEMPO) and BHT (2,6-di-tert-butyl-4methylphenol), suggesting that a radical pathway might be involved in these transformations (Scheme 7). 


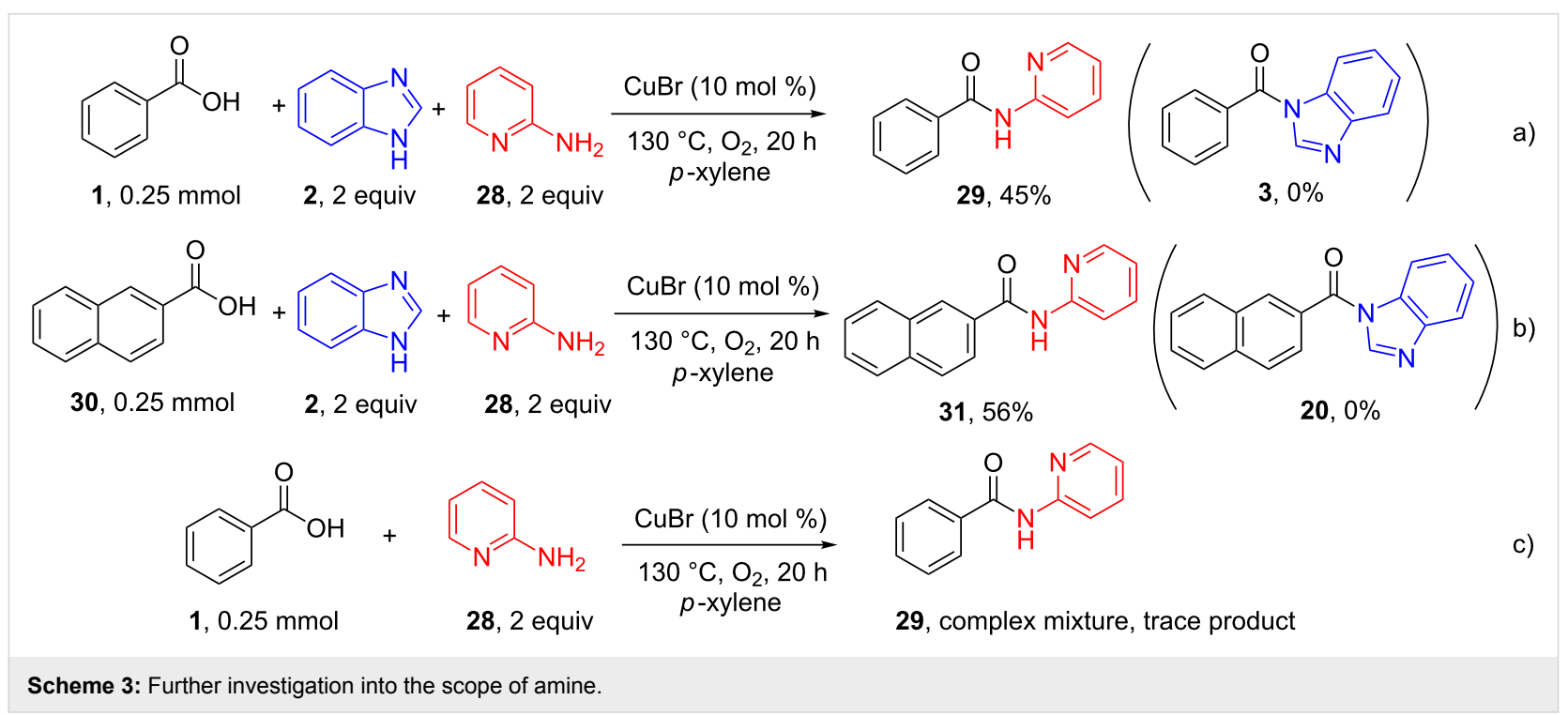

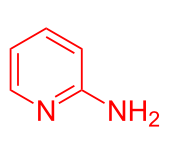

28, 2 equiv

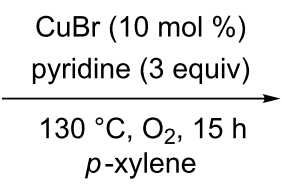<smiles>O=C(Nc1ccccn1)c1ccccc1</smiles>

$29,92 \%$

Scheme 4: Possible transamidation process

A careful look at the literature reveals that $\mathrm{Cu}$ (II) superoxide species were reported to be formed under our standard conditions and might act as a nucleophile to attack carbonyl groups in certain cases [30], although it was always reported as an electrophile [32]. Thus, a new intermediate, peroxycarboxylate, will be formed in our case. In order to figure out whether peroxycarboxylate acts as our key intermediate, the readily available $m$-CPBA (41) was used as substrate under the standard conditions and the corresponding product 19 was obtained in 54\% yield (Scheme 8a).

Compared to the isolated yield from $m$-chlorophenylacetic acid (Scheme 2, 19, 51\%), we have reason to believe that the above proposed mechanism is plausible. In addition, pyridine $N$-oxide was applied to the benzoic acid standard conditions in the presence/absence of dioxygen. However, only a trace amount of the desired product 3 was ever detected, thus it was ruled out as a possible pathway (Scheme $8 \mathrm{~b}$ ).

Based on the above control experiments, we postulated a tentative mechanism (Scheme 9). The copper catalyst was postulated to play dual roles in the initial activation of benzoic acid: (1) it acts as a Lewis acid to activate benzoic acid (1), making it vulnerable to nucleophilic attack and (2) it forms $\mathrm{Cu}(\mathrm{II})$ super- oxide species [30,32-34] to attack activated benzoic acid $\mathbf{A}$ to afford intermediate $\mathbf{B}$. Under the standard conditions, intermediate $\mathbf{B}$ was switched to intermediate $\mathbf{C}$, once again, with the nucleophilic attack from benzimidazole (2) and eventually intermediate $\mathbf{C}$ was converted into the desired $\mathbf{3}$ via intermediate $\mathbf{D}$ along with the recycling of the $\mathrm{Cu}$ catalyst.

\section{Conclusion}

In conclusion, $\mathrm{Cu}$-catalyzed oxidative direct amidation from nonactivated carboxylic acid with benzimidazoles under dioxygen atmosphere with molecular oxygen as an activating reagent has been described. Azole amides were obtained in good yield in an oxidative protocol with a very broad range of substrates. Subsequent transamidation could be performed using the prepared azole amides and a variety of amines. This reaction has many advantages, in particular the use of an inexpensive copper salt as the catalyst, the use of oxygen as both the sole terminal oxidant and activating reagent, and inexpensive and readily available starting materials. This is the first $\mathrm{Cu}$-catalyzed, direct, amide formation between nonactivated carboxylic acids and benzimidazoles in coupling reagent, traditional activating, reagent-free conditions. A mechanistic study demonstrated that a peroxycarboxylate was the key intermediate. This was the first reported example in such a simple system 


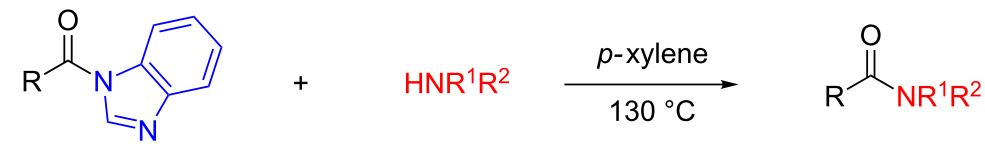<smiles>O=C(Nc1ccccn1)c1ccccc1</smiles>

$29,92 \%$<smiles>O=C(c1ccccc1)n1nnc2ccccc21</smiles>

33, $56 \%$<smiles>O=C(c1ccccc1)n1ccc(-c2ccccc2)n1</smiles>

36, $71 \%$<smiles>CCN(CC)C(=O)c1ccccc1</smiles>

$39,68 \%$<smiles>O=C(Nc1ccccn1)c1ccc2ccccc2c1</smiles>

31, $85 \%$<smiles>O=C(Nc1ccccc1)c1ccccc1</smiles>

$34,68 \%$<smiles>O=C(Nc1ccccn1)C1CCCCC1</smiles>

$37,86 \%$<smiles>O=C(c1ccccc1)n1ncc2ccccc21</smiles>

$32,95 \%$<smiles>CN(Cc1ccccc1)C(=O)c1ccccc1</smiles>

$35,78 \%$<smiles>O=C(c1ccccc1)N1CCCCC1</smiles>

$38,74 \%$

Scheme 5: Scope of the amine transamidation from benzimidazole amides. Reaction conditions: benzimidazole amide $(0.25 \mathrm{mmol})$, amine $(0.375 \mathrm{mmol}), p$-xylene $(1 \mathrm{~mL}), 130^{\circ} \mathrm{C}$, sealed tube. Isolated yields. ${ }^{a}$ Methyl L-leucinate hydrochloride as the ammonia source and $\mathrm{Et}_{3} \mathrm{~N}(1.5$ equiv) were added, the mixture was stirred at room temperature for $3 \mathrm{~min}$, then the mixture was heated to $130^{\circ} \mathrm{C}$.<smiles>O=C(O)c1ccccc1</smiles>

$1,5 \mathrm{mmol}$<smiles>Fc1ccc2[nH]cnc2c1</smiles>

2<smiles>O=C(c1ccccc1)n1cnc2ccccc21</smiles>

Scheme 6: Preparative scale of the reaction.<smiles>O=C(O)c1ccccc1</smiles>

1
$3,65 \%(720 \mathrm{mg})$

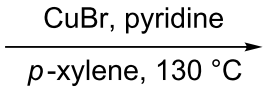


<smiles>COC(=O)c1cccc(Cl)c1</smiles>

1<smiles>c1cnc2[nH]c3cccn3c2c1</smiles>

2<smiles>c1ccc2[nH]cnc2c1</smiles>

2<smiles>O=C(c1cccc(Cl)c1)n1cnc2ccccc21</smiles>

$19,54 \%$

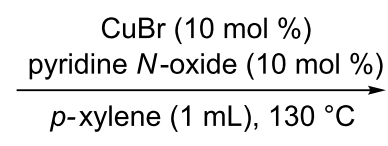

p-xylene $(1 \mathrm{~mL}), 130^{\circ} \mathrm{C}$<smiles>O=C(c1ccccc1)n1cnc2ccccc21</smiles>

3, $4 \%$ (under $\mathrm{O}_{2}$ )

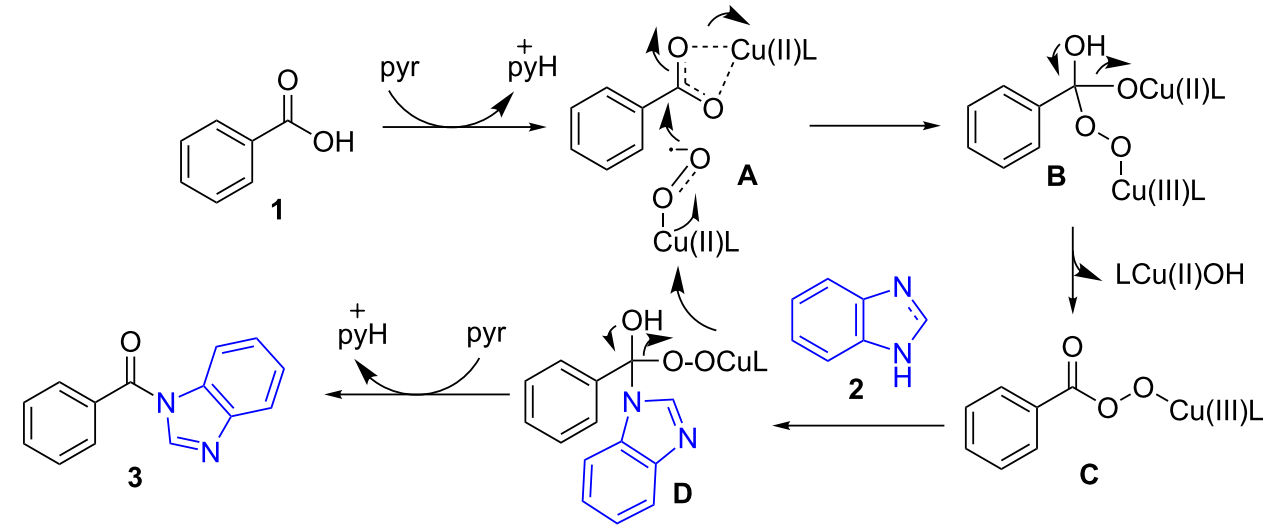

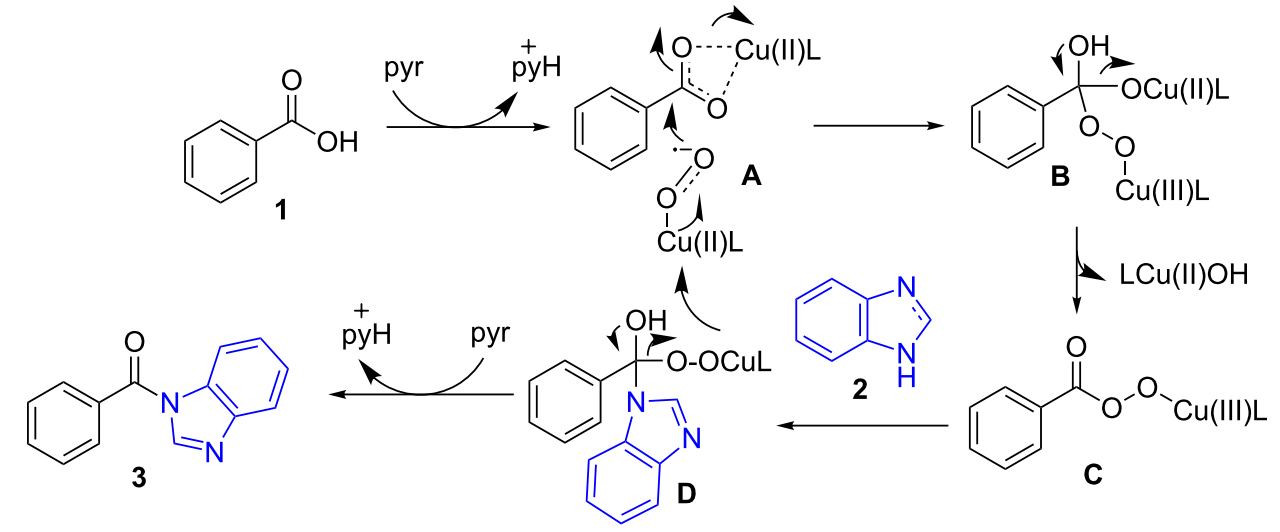

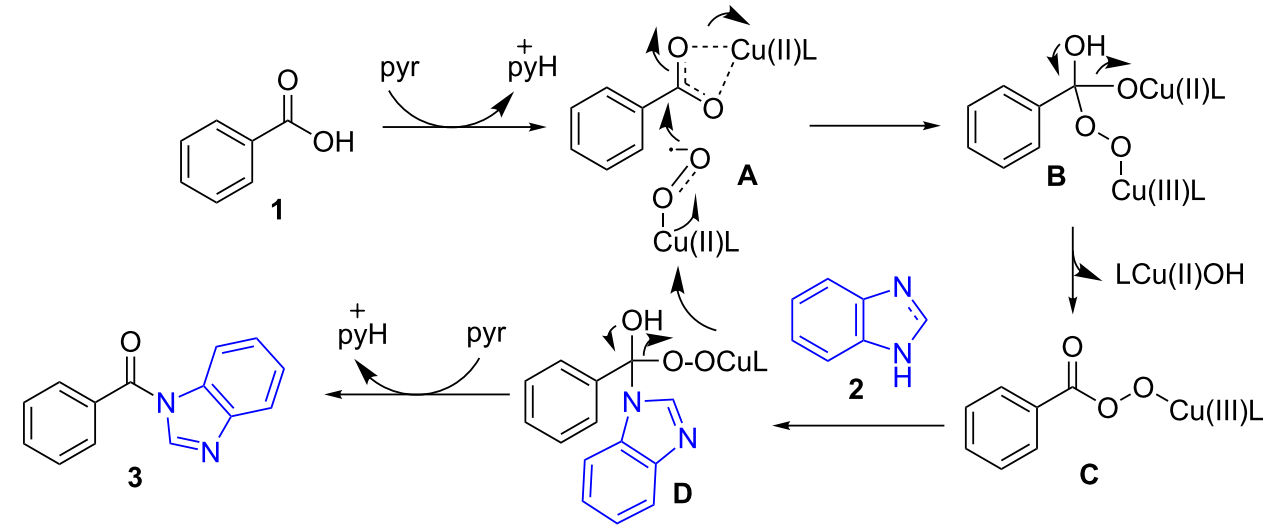

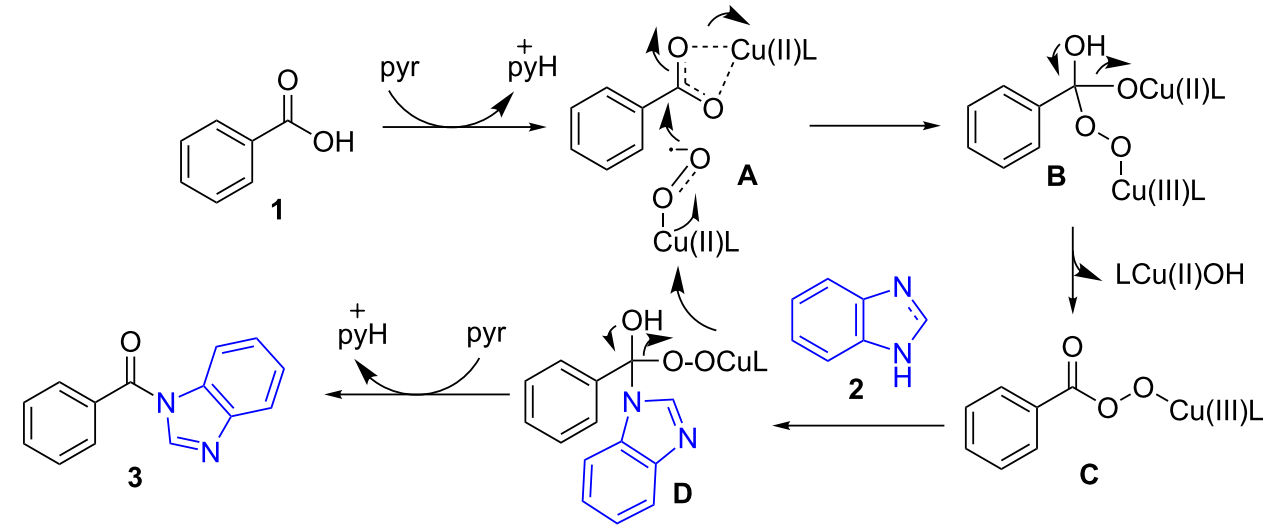

Scheme 9: Proposed mechanism

which could be employed in amide formation. Notably, the transamidation occurred smoothly between the azole amide and a variety of amines, thus providing versatile amides with our new strategy. Therefore, this is a very general method for azole amide formation from versatile carboxylic acids with potential application in organic synthesis.

\section{Supporting Information}

\section{Supporting Information File 1}

Experimental procedures, analytical data and NMR spectra. [http://www.beilstein-journals.org/bjoc/content/ supplementary/1860-5397-11-233-S1.pdf]

\section{Acknowledgements}

Financial support from the National Natural Science Foundation of China (21202049), the Recruitment Program of Global Experts (1000 Talents Plan), Fujian Hundred Talents Program and Program of Innovative Research Team of Huaqiao University are gratefully acknowledged.

\section{References}

1. Humphrey, M. J.; Chamberlin, R. A. Chem. Rev. 1997, 97, 2243-2266. doi:10.1021/cr950005s

2. Valeur, E.; Bradley, M. Chem. Soc. Rev. 2009, 38, 606-631. doi:10.1039/B701677H

3. Mitchell, J. A.; Reid, E. E. J. Am. Chem. Soc. 1931, 53, 1879-1883. doi:10.1021/ja01356a037

4. Shepard, E. E.; Porter, H. D.; Noth, J. F.; Simmans, C. K. J. Org. Chem. 1952, 17, 568-576. doi:10.1021/jo01138a009

5. Schmidt, U.; Dietsche, M. Angew. Chem., Int. Ed. Engl. 1982, 21, 143-144. doi:10.1002/anie.198201431

6. Cheung, C. W.; Fung, H. S.; Lee, S. Y.; Qian, Y. Y.; Chan, Y. W.; Chan, K. S. Organometallics 2010, 29, 1343-1354. doi:10.1021/om9008668

7. Das, S.; Biswas, R.; Mukherjee, B. J. Phys. Chem. B 2015, 119, 274-283. doi:10.1021/jp509869d

8. Yoo, W.-J.; Li, C.-J. J. Am. Chem. Soc. 2006, 128, 13064-13065. doi:10.1021/ja064315b

9. Ekoue-Kovi, K.; Wolf, C. Org. Lett. 2007, 9, 3429-3432. doi:10.1021/ol7014626

10. Reddy, K. R.; Maheswari, C. U.; Venkateshwar, M.; Kantam, M. L. Eur. J. Org. Chem. 2008, 3619-3622. doi:10.1002/ejoc.200800454 
11. Ghosh, S. C.; Ngiam, J. S. Y.; Chai, C. L. L.; Seayad, A. M.; Dang, T. T.; Chen, A. Adv. Synth. Catal. 2012, 354, 1407-1412. doi:10.1002/adsc.201200020

12. Prasad, V.; Kale, R. R.; Mishra, B. B.; Kumar, D.; Tiwari, V. K. Org. Lett. 2012, 14, 2936-2939. doi:10.1021/ol3012315

13. Hosseini-Sarvari, M.; Sharghi, H. J. Org. Chem. 2006, 71, 6652-6654. doi:10.1021/jo060847z

14. Ishihara, K.; Ohara, S.; Yamamoto, H. J. Org. Chem. 1996, 61, 4196-4197. doi:10.1021/jo9606564

15. Allen, C. L.; Chhatwal, A. R.; Williams, J. M. J. Chem. Commun. 2012, 48, 666-668. doi:10.1039/C1CC15210F

16. Goh, K. S.; Tan, C.-H. RSC Adv. 2012, 2, 5536-5538. doi:10.1039/C2RA20773G

17. Subramanian, P.; Indu, S.; Kaliappan, K. P. Org. Lett. 2014, 16, 6212. doi:10.1021/ol5031266

18. Starkov, P.; Sheppard, T. D. Org. Biomol. Chem. 2011, 9, 1320-1323. doi:10.1039/C0OB01069C

19. Tan, B.; Toda, N.; Barbas, C. F., III. Angew. Chem., Int. Ed. 2012, 51, 12538-12541. doi:10.1002/anie.201205921

20. Charville, H.; Jackson, D.; Hodges, G.; Whiting, A. Chem. Commun. 2010, 46, 1813-1823. doi:10.1039/B923093A

21. Kosal, A. D.; Wilson, E. E.; Ashfeld, B. L. Chem. - Eur. J. 2012, 18, 14444-14453. doi:10.1002/chem.201201773

22. Dunlap, F. L. J. Am. Chem. Soc. 1902, 24, 758-763. doi:10.1021/ja02022a008

23. Sheehan, J. C.; Hess, G. P. J. Am. Chem. Soc. 1955, 77, 1067-1068. doi:10.1021/ja01609a099

24. Carey, J. S.; Laffan, D.; Thomson, C.; Williams, M. T. Org. Biomol. Chem. 2006, 4, 2337-2347. doi:10.1039/B602413K

25. Punniyamurthy, T.; Velusamy, S.; Iqbal, J. Chem. Rev. 2005, 105, 2329-2364. doi:10.1021/cr050523v

26. Wang, T.; Jiao, N. J. Am. Chem. Soc. 2013, 135, 11692-11695. doi:10.1021/ja403824y

27. Ley, S. V.; Thomas, A. W. Angew. Chem., Int. Ed. 2003, 42, 5400-5449. doi:10.1002/anie.200300594

28. Bi, H.-P.; Zhao, L.; Liang, Y.-M.; Li, C.-J. Angew. Chem., Int. Ed. 2009, 48, 792-795. doi:10.1002/anie.200805122

29. Liu, J.; Liu, Q.; Yi, H.; Qin, C.; Bai, R.; Qi, X.; Lan, Y.; Lei, A. Angew. Chem., Int. Ed. 2014, 53, 502-506. doi:10.1002/anie.201308614

30. Pirovano, P.; Magherusan, A. M.; McGlynn, C.; Ure, A.; Lynes, A.; McDonald, A. R. Angew. Chem., Int. Ed. 2014, 53, 5946-5950. doi:10.1002/anie.201311152

31. Horton, D. A.; Bourne, G. T.; Smythe, M. L. Chem. Rev. 2003, 103, 893-930. doi:10.1021/cr020033s

32. Yoon, J.; Solomon, E. I. J. Am. Chem. Soc. 2007, 129, 13127-13136. doi:10.1021/ja073947a

33. Kieber-Emmons, M. T.; Ginsbach, J. W.; Wick, P. K.; Lucas, H. R.; Helton, M. E.; Lucchese, B.; Suzuki, M.; Zuberbühler, A. D.; Karlin, K. D.; Solomon, E. I. Angew. Chem., Int. Ed. 2014, 53, 4935-4939. doi:10.1002/anie.201402166

34. Bonnot, F.; Tremey, E.; von Stetten, D.; Rat, S.; Duval, S.; Carpentier, P.; Clemancey, M.; Desbois, A.; Nivière, V. Angew. Chem., Int. Ed. 2014, 53, 5926-5930. doi:10.1002/anie.201400356

\section{License and Terms}

This is an Open Access article under the terms of the Creative Commons Attribution License

(http://creativecommons.org/licenses/by/2.0), which permits unrestricted use, distribution, and reproduction in any medium, provided the original work is properly cited.

The license is subject to the Beilstein Journal of Organic Chemistry terms and conditions:

(http://www.beilstein-journals.org/bjoc)

The definitive version of this article is the electronic one which can be found at:

doi: $10.3762 /$ bjoc. 11.233 